\title{
Synthesis and factorial design applied to a novel chitosan/sodium polyphosphate nanoparticles via ionotropic gelation as an RGD delivery system
}

\author{
Charlene Priscila Kiillla, ${ }^{a}$, Hernane da Silva Barud ${ }^{\mathrm{b}}$, Sílvia Helena Santagneli ${ }^{\mathrm{c}}$, \\ Sidney José Lima Ribeiro ${ }^{\mathrm{c}}$, Amélia M. Silva ${ }^{\mathrm{d}}$, Agnieszka Tercjak ${ }^{\mathrm{e}}$, Junkal Gutierrez ${ }^{\mathrm{e}}$, \\ Andressa Maria Pironi ${ }^{\mathrm{b}}$, Maria Palmira Daflon Gremião ${ }^{\mathrm{a}}$ \\ a Univ. Estadual Paulista, Faculty of Pharmaceutical Sciences, Rod. Araraquara-Jau, Km 1, 14802-901, Araraquara, SP, Brazil \\ b Laboratório de Biopolímeros e Biomateriais (BioPolMat) - Centro Universitário de Araraquara (UNIARA), Araraquara, SP, Brazil \\ c Institute of Chemistry, UNESP - Univ. Estadual Paulista, Rua Prof. Francisco Degni, 55, 14800-900, Araraquara, SP, Brazil \\ d Department of Biology and Environment, Centre for Research and Technology of Agro Environmental and Biological Sciences (CITAB/UTAD), University of \\ Trás-os-Montes e Alto Douro (UTAD), P.O. Box 1013, 5001-801, Vila Real, Portugal \\ ' Group 'Materials + Technologies' (GMT), Department of Chemical and Environmental Engineering, Engineering College of Gipuzkoa, University of the \\ Basque Country (UPV/EHU), Plaza Europa 1, 20018 Donostia-San Sebastián, Spain
}

\section{A R T I C L E I N F O}

\section{Article history:}

Received 20 July 2016

Received in revised form 18 November 2016

Accepted 18 November 2016

Available online 21 November 2016

\section{Keywords:}

Chitosan

Nanoparticles

Graham's salt

Ionic gelation

Experimental factorial design

cRGDfV

\begin{abstract}
A B S T R A C T
Chitosan nanoparticles have been extensively studied for both drug and protein/peptide delivery. The aim of this study was to develop an optimized chitosan nanoparticle, by ionotropic gelation method, using $3^{2}$ full factorial design with a novel polyanion, sodium polyphosphate, well known under the trade name Graham salt. The effects of these parameters on the particle size, zeta potential, and morphology and association efficiency were investigated. The optimized nanoparticles showed an estimated size of $166.20 \pm 1.95 \mathrm{~nm}$, a zeta potential of $38.7 \pm 1.2 \mathrm{mV}$ and an efficacy of association of $97.0 \pm 2.4 \%$. The Atomic Force Microscopy (AFM) and Scanning Electronic Microscopy (SEM) revealed spherical nanoparticles with uniform size. Molecular interactions among the components of the nanoparticles and peptide were evaluated by Fourier Transform Infrared Spectra (FTIR) and Differential Scanning Calorimetry (DSC). The obtained results indicated that, the developed nanoparticles demonstrated high biocompatible, revealing no or low toxicity in the human cancer cell line (Caco-2). In conclusion, this work provides parameters that contribute to production of chitosan nanoparticles and sodium polyphosphate with desirable size, biocompatible and enabling successful use for protein/peptides delivery.
\end{abstract}

(C) 2016 Elsevier Ltd. All rights reserved.

\section{Introduction}

Chitosan (Ch) is a polysaccharide composed of $\beta$-(1-4)-linked D-glucosamine and $N$-acetyl-D-glucosamine. In particular, Ch has advantageous biological properties, such as relative non-toxicity, biodegradability, biocompatibility and bioadhesive characteristics. Chitosan-based nanoparticles have been extensively studied for delivery of therapeutic proteins and peptides (1990; Fundueanu et al., 2004; Gavini et al., 2006; Gavini, Rassu, Sanna, Cossu, \& Giunchedi, 2005). Ch have positively charged surfaces in biologi-

\footnotetext{
* Corresponding author at: Faculdade de Ciências Farmacêuticas, Univ. Estadual Paulista - UNESP, Rodovia Araraquara-Jau, Km 1, CEP, 14801-902, Araraquara, SP, Brazil.

E-mail address: char_kiill@yahoo.com.br (C.P. Kiilll).
}

cal fluid, thus various macromolecular drugs containing negatively charged ones (e.g., peptides, proteins and genes) can be incorporated by the electrostatic interactions for their delivery into the site of action (Calvo, Remuñán-López, Vila-Jato, \& Alonso, 1997; Csaba, Koping-Hoggard, \& Alonso, 2009; Sadeghi et al., 2008).

Among the variety of methods developed to prepare $\mathrm{Ch}$ nanoparticles, ionotropic gelation technique has attracted attention since nanoparticles obtained by this process are non-toxic, it requires mild control conditions without involving high temperatures and is organic solvent free. All previously mentioned characteristics are important to preserve the protein/peptide structure and function during the nanoparticles formation (Chandra Hembram, Prabha, Chandra, Ahmed, \& Nimesh, 2016; Chen, Zhang, \& Huang, 2007). 
Ionotropic gelation technique is based on the ionic interactions between the positively charged $\mathrm{Ch}$ and the negatively charged groups of polyanions, such as sodium tri-polyphosphate, which is the most extensively used ion crosslinking due to its non-toxic and multivalent properties (Fan, Yan, Xu, \& Ni, 2012). However, other work proposed a novel polyanion, composed of long metaphosphate chains, sodium polyphosphate. It is the only water soluble inorganic polyphosphate able to spontaneously form nanoparticles by electrostatic interactions between the long polyphosphate chains and the Ch (Casettari, Cespi, Palmieri, \& Bonacucina, 2013; Pickup et al., 2014).

In this work, we choose a class of macromolecules called disintegrins that comprise a group of low molecular weight proteins that interact with integrin receptors on the surface of cancer cells. Pertaining to a family of cysteine-rich proteins isolated from snake venoms, these peptides are known to inhibit cell-to-matrix and cell-to-cell interactions mediated by integrins.

However, administration of therapeutic disintegrins is constrained mainly by their low bioavailability and susceptibility to acidic and enzymatic degradation in biological fluids. In order to circumvent problems related to drug stability and consequent loss of activity, researchers have begun exploring novel protein drug delivery systems, e.g., liposomes, microparticles and nanoparticles (Andreani, Kiill et al., 2014; Andreani, de Souza et al., 2014; Callens \& Remon, 2000; Chen et al., 2007; Dudhani \& Kosaraju, 2010; Liu, Xu, Jiang, \& Yuan, 2012). Among them, polymeric nanoparticles, such as chitosan nanoparticles, stand out as a promising delivery strategy for therapeutic protein/peptides (Dudhani \& Kosaraju, 2010; Fundueanu et al., 2004; Gan, Wang, Cochrane, \& McCarron, 2005; Gavini et al., 2006).

In the present study, we focused on development of chitosan nanoparticles (Ch-NPs) using a novel crosslinking agent, sodium polyphosphate (PP). The aim of this study was the preparation and characterization of chitosan/polyphosphate nanoparticles (Ch-PP-NPs) that represent promising carrier properties for proteins/peptides delivery. The developed chitosan nanoparticles, prepared by the ionotropic gelation method using sodium polyphosphate as a crosslinking agent, were characterized by different techniques to determinate the interaction between components of prepared NPs and their size. The biocompatibility of the Ch-PP-NPs and cRGDfV-loaded Ch-PP-NPs were performed on Caco-2 cell line.

\section{Materials and methods}

\subsection{Materials}

Low molecular weight Chitosan (Ch) was purchased from Sigma-Aldrich (USA) with deacetylation degree of $75 \%-85 \%$. Sodium polyphosphate and acetic acid were purchased from Merck (Germany). Bicinchoninic acid (BCA) solution was purchased from Pierce (Thermo Scientific and Life Science Research Products Rockford, IL USA). cRGDfV (Arginine-Glycine-Aspartic Acid-D-Phenylalanine-Valine) disintegrin was kindly provided as a powder and were purchased from Mocell Biotech Limited (Shangai, China). All other chemicals were at least reagent grade and used as received. Water used was of high purity (Milli-Q, Millipore).

\subsection{Experimental factorial design}

Three different variables and their influence on the physicochemical properties of chitosan nanoparticles were evaluated using a 9 full factorial design composed of 2 variables which were set at 3-levels each. Independent variables were ratio PP (w/v) and Ch $(\mathrm{w} / \mathrm{v})$. The established dependent variables were the mean parti- cle size (Z-Ave), polydispersity index (PdI) and zeta potential (ZP). The design required a total of 9 experiments. For each factor, the lower and higher values of the lower and upper levels were represented by a lower level $(-1)$, medium and a higher level $(+1)$ sign, respectively (Table 1 ). These were chosen on the basis of the tested lower and upper values for each variable, according to preformulation studies and literature research (de Pinho Neves et al., 2014). A factorial design approach was applied to maximize the experimental efficiency requiring a minimum of experiments to optimize the chitosan nanoparticles produced by ionotropic gelation technique. The data were analyzed using the STATISTICA 10.0 (StafSoft, Inc.) software.

\subsection{Development of the chitosan/polyphosphate nanoparticles}

The Ch-PP-NPs were prepared following the procedure described by Calvo et al. (1997). Ch solution $(2 \mathrm{mg} / \mathrm{mL} ; 3 \mathrm{mg} / \mathrm{mL}$ and $4.4 .1 \mathrm{mg} / \mathrm{mL}$ ) was prepared by dissolving $\mathrm{Ch}$ in a $0.75 \%(\mathrm{v} / \mathrm{v})$ acetic acid solution $(0.1 \mathrm{M})$ and leaving it under stirring for $24 \mathrm{~h}$. The $\mathrm{pH}$ was adjusted to 4.4 with a $0.5 \mathrm{M}$ sodium hydroxide solution and diluted in deionized water to the final desired concentrations. PP was dissolved in deionized water to a final concentration of $1 \mathrm{mg} / \mathrm{mL}$. Then, the PP solution was added to the Ch solution dropwise at different $\mathrm{Ch} / \mathrm{PP}$ ratios under vigorous magnetic stirring at room temperature (Calvo et al., 1997). cRGDfV-loaded Ch-PP-NPs were prepared by dissolving the cRGDfV $(1 \mathrm{mg} / \mathrm{mL})$ in the PP solution which was then added to $\mathrm{Ch}$ solution dropwise under magnetic stirring. The indicated nomenclature for produced nanoparticles has in account the quantity of $\mathrm{Ch}$ and of PP used in the respective formulation, and a complete list is shown in Table 2.

\subsection{Physicochemical characterization}

\subsubsection{Mean particle size and zeta potential analysis}

Particles size (Z-Ave), polydispersity index (PdI) and zeta potential (ZP) were determined by using a dynamic light scattering technique (Zetasizer model Nano ZS, Malvern Instruments, UK) with red laser of $633 \mathrm{~nm}$. All samples were diluted with ultra purifiedwater to suitable concentration and analyzed in triplicate. ZP of nanoparticles andcRGDfV-loaded Ch-PP-NPs were also measured with the same instrument since it allows determining the electrophoretic mobility to assess the surface electrical charge of the nanoparticles.

\subsubsection{Fourier Transmission Infrared Spectroscopy (FTIR)}

Ch-PP-NPs were separated from suspension and were dried by a freeze-dryer (Thermo ${ }^{\circledR}$ - MicroModulyo115), then were gently mixed with a suitable amount of micronized $\mathrm{KBr}$ powder to prepare the $\mathrm{KBr}$ pellets. Infrared spectra were performed using a FTIR spectrometer (Shimadzu ${ }^{\circledR}$ Europe - Prestige-21) with resolution of $4 \mathrm{~cm}^{-1}$ and 128 scans.

\subsubsection{Differential scanning calorimetry (DSC)}

DSC analysis was carried out in a Mettler DSC apparatus (Mettler Toledo, Gieben, Switzerland). The instrument was calibrated with indium and zinc and DSC scans were recorded from $25^{\circ} \mathrm{C}$ to $350^{\circ} \mathrm{C}$ at a heating constant rate of the $10^{\circ} \mathrm{C} / \mathrm{min}$ under a nitrogen purge $\left(50 \mathrm{~mL} \mathrm{~min}^{-1}\right)$. The DSC parameters, including enthalpy and onset temperature, were calculated by the STARe Software.

\subsubsection{Morphological characterization}

The morphological examination of the Ch-PP-NPs was determined by scanning electron microscopy (SEM) (Jeol JSM 7500 F) and using atomic force microscopy (AFM). For SEM analysis the nanoparticles were prepared by placing a drop of colloid dispersion containing nanoparticles on a sample holder and then coated 
Table 1

Initial $3^{2}$ full factorial design, providing the lower $(-1)$, medium level (0) and upper (+1) level values for each variable.

\begin{tabular}{|c|c|c|c|c|}
\hline Dependent variables & Lower level $(-1)$ & Medium level (0) & Higher level (+1) & Independent variables \\
\hline Chitosan\% (w/v) & 2.0 & 3.0 & 4.41 & Z-Ave; PdI and ZP \\
\hline Polyphosphate\% (w/v) & 0.6 & 1.2 & 2.1 & \\
\hline
\end{tabular}

Table 2

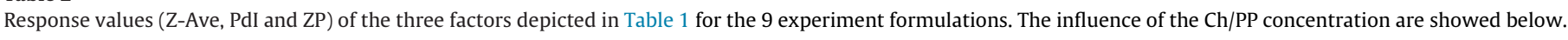

\begin{tabular}{|c|c|c|c|c|c|c|}
\hline \multirow[t]{2}{*}{ Nanoparticles } & \multicolumn{3}{|l|}{ Variable } & \multicolumn{3}{|l|}{ Responses } \\
\hline & $\mathrm{Ch} \%(\mathrm{w} / \mathrm{v})$ & $\mathrm{PP} \% \mathrm{w} / \mathrm{v})$ & Ratio Ch:PP & Z-Ave (nm) \pm SD & $\mathrm{PdI} \pm \mathrm{SD}$ & $\mathrm{PZ} \pm \mathrm{SD}$ \\
\hline Ch2PP6-NPs & $(-) 2.0$ & $(-) 0.6$ & 3.3:1 & $166.20 \pm 1.95$ & $0.283 \pm 0.02$ & $38.7 \pm 1.20$ \\
\hline Ch2PP12-NPs & $(-) 2.0$ & (0) 1.2 & $1.6: 1$ & $20.10 \pm 2.95$ & $0.364 \pm 0.04$ & $35.0 \pm 2.30$ \\
\hline Ch2PP21-NPs & $(-) 2.0$ & $(+) 2.1$ & $0.95: 1$ & $336.0 \pm 44.0$ & $0.417 \pm 0.03$ & $32.0 \pm 3.10$ \\
\hline Ch3PP6-NPs & (0) 3.0 & $(-) 0.6$ & $5: 1$ & $316.0 \pm 14.9$ & $0.288 \pm 0.07$ & $38.1 \pm 1.32$ \\
\hline Ch3PP12-NPs & (0) 3.0 & (0) 1.2 & $2.5: 1$ & $424.50 \pm 21.4$ & $0.433 \pm 0.03$ & $33.0 \pm 2.10$ \\
\hline Ch3PP21-NPs & (0) 3.0 & $(+) 2.1$ & $1.4: 1$ & $431.60 \pm 8.79$ & $0.466 \pm 0.11$ & $31.0 \pm 1.45$ \\
\hline Ch441PP6-NPs & $(+) 4.41$ & $(-) 0.6$ & 7.3:1 & $472.50 \pm 9.79$ & $0.314 \pm 0.02$ & $32.5 \pm 2.54$ \\
\hline Ch441PP12-NPs & $(+) 4.41$ & (0) 1.2 & $3.6: 1$ & $534.80 \pm 10.1$ & $0.489 \pm 0.166$ & $30.2 \pm 2.40$ \\
\hline Ch441PP21-NPs & $(+) 4.41$ & (+) 2.1 & $2.1: 1$ & $562.40 \pm 14.1$ & $0.502 \pm 0.02$ & $29.5 \pm 3.40$ \\
\hline
\end{tabular}

$\mathrm{Z}$-Ave = mean particle size; $\mathrm{PdI}=$ polydispersity index; $\mathrm{ZP}=$ zeta potential.

with a thin layer of carbon and examined by electron microscope at an intensity of $2.0 \mathrm{kV}$.

In the case of AFM, all the nanoparticles were spin-coated onto silicon wafer substrates using a spin-coater (Model P6700 from Specialty Coating Systems, Inc.) at $2000 \mathrm{rpm}$ for $120 \mathrm{~s}$. Prepared samples were analyzed by AFM operating in a tapping mode with a scanning probe microscope Dimension ICON from Bruker equipped with an integrated silicon tip/cantilever having a resonance frequency of $300 \mathrm{kHz}$. Scan rates ranged from 0.7 to $1.2 \mathrm{~Hz} \mathrm{~s}^{-1}$. In order to obtain repeatable results, different regions of the specimens were scanned to choose representative AFM images. Taking into account that obtained height and phase AFM images were very similar, only AFM phase images are shown.

\subsection{Association efficiency}

The amount of cRGDfV associated into the Ch-PP-NPs was determined in the supernatant using the bicinchoninic acid (BCA) colorimetric assay (Sigma-Aldrich Co., St. Louis, Missouri). The amount of cRGDfV-loaded in the nanoparticles was calculated as the difference between the peptide loaded and the peptide recovered in the supernatant. The supernatant of unloaded nanoparticles was used as a blank, in order to subtract the interference of the components of loaded samples with the BCA. The assay was realized in triplicate for each batch. Association Efficiency (AE) was calculated using the following equation:

$\frac{A E(\%)=\text { total amount of } c R G D f V-\text { free } c R G D f V}{\text { total amount of } c R G D f V} \times 100$

\subsection{In vitro assay}

\subsubsection{Cell line}

The cells used in this work were obtained from American Type Culture Collection (ATCC - Rockville, Maryland, USA), namely, the human epithelial colorectal adenocarcinoma Caco-2 (ATCC ${ }^{\circledR}$ HTB-37 $7^{\mathrm{TM}}$ ) cell line. The cells were maintained in appropriated medium (Dulbecco's Modified Eagle Medium (DMEM), supplemented with $10 \%(\mathrm{v} / \mathrm{v}$ ) fetal bovine serum (FBS), 2 mM L-glutamine, and antibiotics ( $50 \mathrm{U} \mathrm{mL}^{-1}$ penicillin, $100 \mu \mathrm{g} \mathrm{mL}^{-1}$ of streptomycin and $0.25 \mu \mathrm{g} / \mathrm{mL}$ amphotericin B), all items obtained from Gibco, Alfagene, Invitrogen, Portugal) at $37^{\circ} \mathrm{C}$, in an atmosphere of $5 \% \mathrm{CO}_{2}$ and controlled humidity. In this study, cells were used between passages 41 and 46 .

\subsubsection{Measurement of Caco-2 cells viability uppon exposure to} cRGDfV-loaded Ch-PP-NPS

The cell viability assay used in this study consisted on the Alamar Blue ${ }^{\circledR}$ assay. This method consists on the conversion of resazurin (Bluebond-Langner, Perkel, Goertzel, Nelson, \& McGeary, 1990) into resorufin (pink/red), whose absorbance can be determined spectrophotometrically (Bluebond-Langner et al., 1990). This method provides a quantitative measure of the number of viable cells that continuously convert resazurin to resorufin, and thus producing a quantitative measure of cell viability (or cytotoxicity). After trypsin treatment, Caco-2 cells were counted and diluted in culture medium (at density of $10^{5}$ cells $/ \mathrm{mL}$ ) and then were seeded in 96 -well plates $(100 \mu \mathrm{L} /$ well). Then, $24 \mathrm{~h}$ after seeding, the culture media was removed and replaced by media containing Ch-PP-NPs, cRGDfV solution and cRGDfV-loaded ChPP-NPs were added at different concentrations $(1,5,10,15$ and $20 \mu \mathrm{g} / \mathrm{mL}$ ). Microplates were placed in the incubator, and cells were exposed for $24 \mathrm{~h}$ and $48 \mathrm{~h}$ at $37^{\circ} \mathrm{C}$ and $5 \% \mathrm{CO}_{2}$. The Alamar Blue assay was conducted according to the manufacturer's protocol. The absorbance readings occurred about $4 \mathrm{~h}$ after Alamar Blue addition, at 570 and $620 \mathrm{~nm}$ using a Multiskan EX microplate reader (MTX Labsystems, USA). The percentage of Alamar Blue reduction was calculated according to the following equation:

$\% A B$ reduction $=\frac{\left(\varepsilon_{o x} \lambda_{2}\right)\left(A \lambda_{1}\right)-\left(\varepsilon_{o x} \lambda_{1}\right)\left(A \lambda_{2}\right)}{\left(\varepsilon_{r e d} \lambda_{1}\right)\left(A^{\prime} \lambda_{2}\right)-\left(\varepsilon_{r e d} \lambda_{2}\right)\left(A^{\prime} \lambda_{1}\right)} \times 100$

In the formula, $\varepsilon \lambda_{2}$ and $\varepsilon \lambda_{1}$ are constants representing the molar extinction coefficient of $A B$ at 620 and $570 \mathrm{~nm}$, respectively, in the oxidized $\left(\varepsilon_{o x}\right)$ and reduced $\left(\varepsilon_{\text {red }}\right)$ forms. $A \lambda_{1}$ and $A \lambda_{2}$ represent absorbance of test wells at $570 \mathrm{~nm}$ and $620 \mathrm{~nm}$, respectively. $A^{\prime} \lambda_{2}$ and $A^{\prime} \lambda_{1}$ represent absorbance of negative control wells at 620 and $570 \mathrm{~nm}$, respectively.

\subsection{Statistical analysis}

Data were expressed as mean \pm standard deviation, and were compared by analysis of variance (ANOVA), Tukey-Kramer's and Dunnet's test. Differences were considered statistically significant at $p$ value $<0.05^{*}$. The program used was GraphPad Prism 5.00 (GraphPad Software, San Diego, USA). 


\section{Results and discussion}

\subsection{Experimental factorial design, mean particle size and zeta potential analysis}

To analyze the dependent variables for the development of an optimal and stable Ch-PP-NPs based on an ionotropic gelation process, a $3^{2}$ full factorial design was employed to provide information about the effects of the selected variables (Table 1) on Z-Ave, PdI and $Z P$. The results showed in Table 1 indicate that the ratio between $\mathrm{Ch}$ and PP are critical and this parameter controls the size distribution of the Ch-PP-NPs. This analysis indicates that particle size characteristics is very important due to its strong influence on the biological performance of the nanoparticles (Jain, Thakur, Sharma, Kush, \& Jain, 2016; Jin et al., 2016; Joseph, Sangeetha, \& Gomathi, 2016; Montha, Maneeprakorn, Buatong, Tang, \& Pon-On, 2016; Wang, Tong, Liu, Liu, \& Li, 2015).

The average size range of Ch-PP-NPs were from $166.2 \pm 1.95 \mathrm{~nm}$ for Ch2PP6-NPs to $562.4 \pm 14.1 \mathrm{~nm}$ for Ch441PP21-NPs, whereas PdI ranged from $0.283 \pm 0.02$ to $0.502 \pm 0.002$ (Table 2). The nanoparticles with lower concentration of $\mathrm{Ch}$ are smaller than that of other formulations, which may be due to the effective ratio between Ch:PP generated by the electrostatic interaction between the negative charge of PP and the positive charge of Ch according to the factorial design experimental data.

The other parameter of characterization of nanoparticles is the surface charge of the nanoparticles directly related to zeta potential. All Ch-PP-NPs are positively charged in the range from 30.2 to $38.7 \mathrm{mV}$, which was attributed to the positive charge of the $\mathrm{Ch}$, as shown in Table 2. High positive values of ZP can reflected in luck of aggregation of investigated nanoparticles, and thereby stabilize their dispersion. Thus, according to previous studies (Barbi et al., 2015; Csaba et al., 2009; Fabregas et al., 2013; Hasanovic, Zehl, Reznicek, \& Valenta, 2009; Huang \& Yang, 2004; Jiang, Wu, Xu, Wang, \& Zeng, 2011; Jonassen, Kjoniksen, \& Hiorth, 2012; Koukaras, Papadimitriou, Bikiaris, \& Froudakis, 2012) for Ch-Tripolyphosphate nanoparticles, the Z-Ave and ZP obtained to Ch-PP-NPs are similar to that found in the literature.

In order to evaluate the effect of $\mathrm{Ch}$ and $\mathrm{PP}$ concentration the surface response was plotted in Fig. 1. One can observed a gradual increase in the Z-Ave (Fig. 1) with an increase in the Ch and PP concentration. For each of the 2 variables present in Fig. 1, the analysis of variance (ANOVA) (data not shown) showed that all the variables have a significant effect ( $p$-value $<0.05$ ) on Z-Ave, however, the Ch concentration $(>3.0 \%, \mathrm{w} / \mathrm{v})$ was the most important factor responsible for the increase in the nanoparticles size. This trend may be explained by the fact that higher amounts of Ch may not lead to complete solubilization and thus resulting in an increase in the nanoparticles size or even aggregation. Nanoparticles with lowest particle size $(166.20 \mathrm{~nm})$ were obtained for the lowest $\mathrm{Ch}$ $(2.0 \%, \mathrm{w} / \mathrm{v})$ and $\mathrm{PP}(0.6 \%, \mathrm{w} / \mathrm{v})$ concentration.

This explains the results depicted in the surface responses (Fig. 1A), showing that the amount of $\mathrm{Ch}$ affects the Z-Ave.

As shown in Fig. 1B, the surface respond showed that the PP concentration have a higher influence on the PdI values. When the amount of PP increased from $0.6 \% \mathrm{w} / \mathrm{v}$ to $2.1 \% \mathrm{w} / \mathrm{v}$, PdI values increased significantly ( $p$-value $=0.002$ ). These results confirmed that the fraction of free primary amino groups of Ch decreased with an increase of the PP concentration $(>1.2 \% \mathrm{w} / \mathrm{v})$, indicating decrease in the cross-linking interactions between the amino groups of $\mathrm{Ch}$ and the PP, as a consequence of a decrease of the cross-linking density between $\mathrm{Ch}$ and PP leading an increase in the polydispersity of the nanoparticles (Csaba et al., 2009; Hu et al., 2008; Yang, Fu, Wang, \& He, 2009).

Fig. 1C showed that Ch-PP-NPs developed had a higher and positive $\mathrm{ZP}$ and the surface response graph shows the effect of variables on the ZP and was found to have statistical significance $(p$-value $=0.007)$ when the PP concentration increased.

Since Z-Ave and PdI are the limiting factors, the aim of this factorial design was to optimize a formulation with appropriate physicochemical parameters for the incorporation of the peptide cRGDfV, as a model of therapeutic peptide. Thus, from the obtained results, an optimal Ch-PP-NPs was found to be composed of lower concentration of $\mathrm{Ch}(2.0 \% \mathrm{w} / \mathrm{v})$ and lower concentration of PP $(0.6 \% \mathrm{w} / \mathrm{v})$. Ch2PP6-NPs were thus selected as the optimized nanoparticles formulation for peptide encapsulation. However, the other two Ch-PP-NPs, Ch2PP12-NPs and Ch2PP21-NPs will be characterized as a comparison to understand the influence in the increase the PP concentration on the nanoparticles formation, since this is the first time using a Graham salt to make Ch nanoparticles.

\subsection{Morphological characterization}

The size and shape of Ch2PP6-NPs were examined by SEM and AFM. As can be clearly seen from both measurement the Ch2PP6NPs showed a spherical shape with smooth surfaces (Fig. 2, left) and had a homogeneous particle size distribution in the range of $70-100 \mathrm{~nm}$. The discrepancy in the size of Ch-PP NPs between DLS and SEM can be related to the fact that DLS measurement of Ch-PP NPs was performed in aqueous media and gives a hydrodynamic diameter of nanoparticles, while SEM and AFM gives diameter of nanoparticles in dry form.

AFM phase images of Ch2PP6-NPs are shown in Fig. 2 (right). Similarly, to SEM results, nanoparticles had a spherical shape with the size lower than $100 \mathrm{~nm}$ homogeneously dispersed on the silica wafer surface. Additionally, same aggregates of Ch2PP6-NPs were also distinguished. The aggregates can be a consequence of the sample-drying procedure, arising from the decrease in solvent volume surrounding the nanoparticles. The size of Ch-PP NPs obtained from AFM was slightly smaller than the equivalent size data obtained from DLS analysis probably as consequence of direct tip-nanoparticles interactions in the case of AFM. Deeper analysis of the AFM data indicated the average size of nanoparticles in the ranges of 30-50 $\mathrm{nm}$ (Fig. 2, right). de Moura et al. (2009) found similar results for Ch-Tripolyphosphate nanoparticles developed for the same concentrations as used in this study. Additionally, as visualized in high magnification AFM images, each analyzed nanoparticles was surrounded by softer material which appeared darker in AFM phase images. Thus every single nanoparticles was covered by softer materials with the size of around $5-10 \mathrm{~nm}$.

\subsection{Structural and thermal characterization}

Fig. 3 shows the FTIR spectra relative to the Ch(a), Ch2PP6-NPs at $0.6 \% \mathrm{w} / \mathrm{v}$ of PP (b), Ch2PP12-NPs at $1.2 \% \mathrm{w} / \mathrm{v}$ of PP (c), Ch2PP21-NPs at $2.1 \% \mathrm{w} / \mathrm{v}$ of $\mathrm{PP}(\mathrm{d})$ and $\mathrm{PP}(\mathrm{e})$.

In the FTIR spectrum of $\mathrm{Ch}$, we observe seven main bands which can be attributed to the $\mathrm{O}-\mathrm{H}$ and $\mathrm{N}-\mathrm{H}$ stretch at $3290 \mathrm{~cm}^{-1}, \mathrm{C}-\mathrm{H}$ stretch at $2872 \mathrm{~cm}^{-1}$, the amide I at $1660 \mathrm{~cm}^{-1}$, the $\mathrm{N}-\mathrm{H}$ bending from amine and amide II at $1593 \mathrm{~cm}^{-1},-\mathrm{CH}_{2}$ bending at $1422 \mathrm{~cm}^{-1}$, a symmetrical deformation of $\mathrm{CH}_{3}$ groups at $1380 \mathrm{~cm}^{-1}$ and antisymmetric stretch of $\mathrm{C}-\mathrm{O}-\mathrm{C}$ and $\mathrm{C}-\mathrm{H}$ stretch at $1156 \mathrm{~cm}^{-1}$. For pure $\mathrm{NaPO}_{3}$ compound, the data can be interpreted by considering previous publications about sodium phosphate (Brow, 2000; Brow, Tallant, Myers, \& Phifer, 1995; Hudgens, Brow, Tallant, \& Martin, 1998; Khawaja, Durrani, Al-Adel, Salim, \& Hussain, 2016; Moustafa \& El-Egili, 1998). The FTIR spectra displays bands at $1255 \mathrm{~cm}^{-1}$ and $1160 \mathrm{~cm}^{-1}$, being assigned, respectively, to the asymmetric and to the symmetric stretching vibration modes of $\left(\mathrm{PO}_{2}\right)$ groups of metaphosphate units. In addition, bands at $850 \mathrm{~cm}^{-1}$ and $770 \mathrm{~cm}^{-1}$ are assigned to the asymmetric and symmetric stretching modes of 

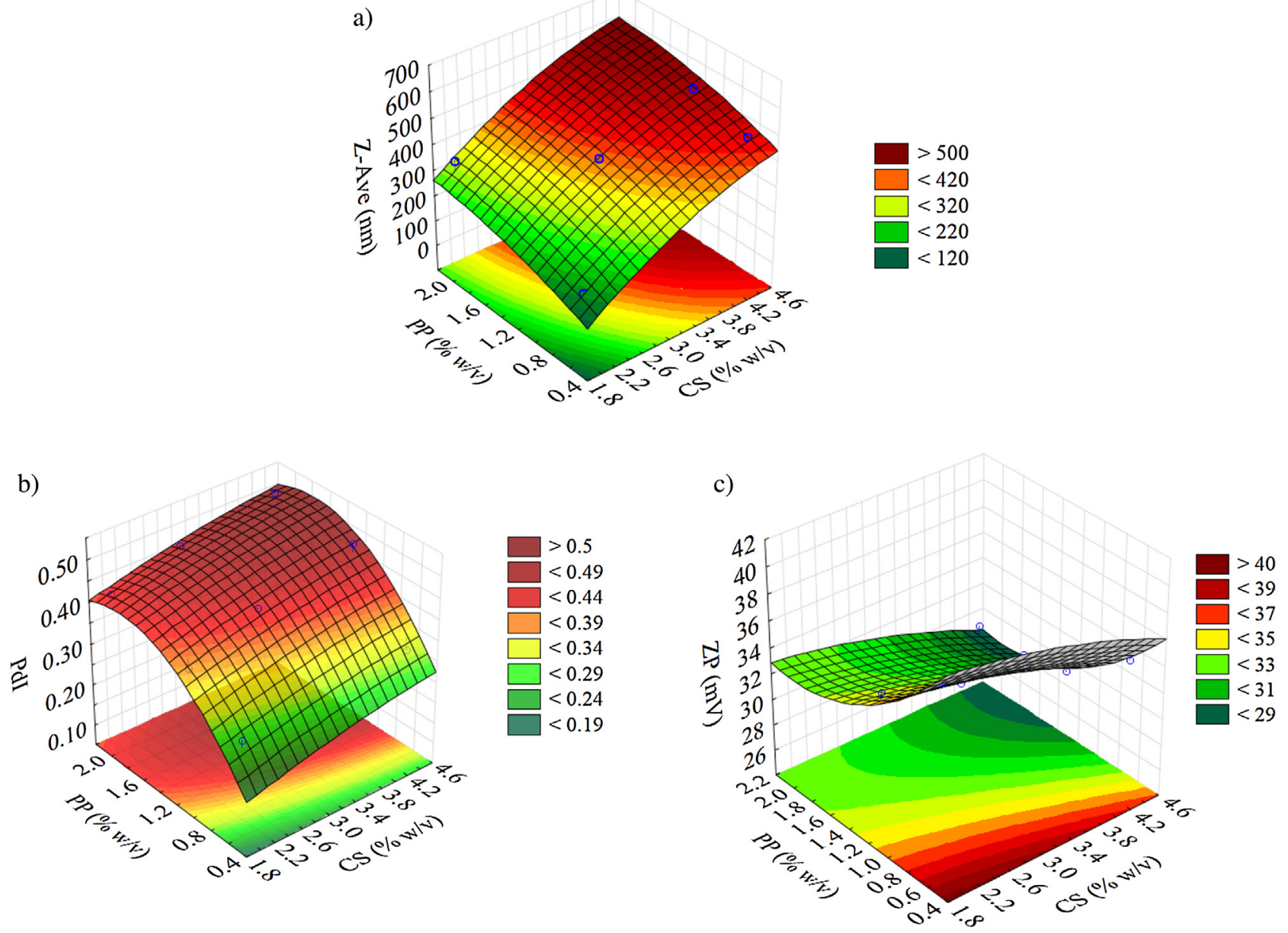

Fig. 1. Surface response chart of: (a) the effect of the concentration (w/v) of Ch and PP on the Z-Ave; (b) the effect of the concentration (w/v) of Ch and PP on the PdI. and (c) the effect of the concentration $(w / v)$ of Ch and PP on the ZP.

(a)
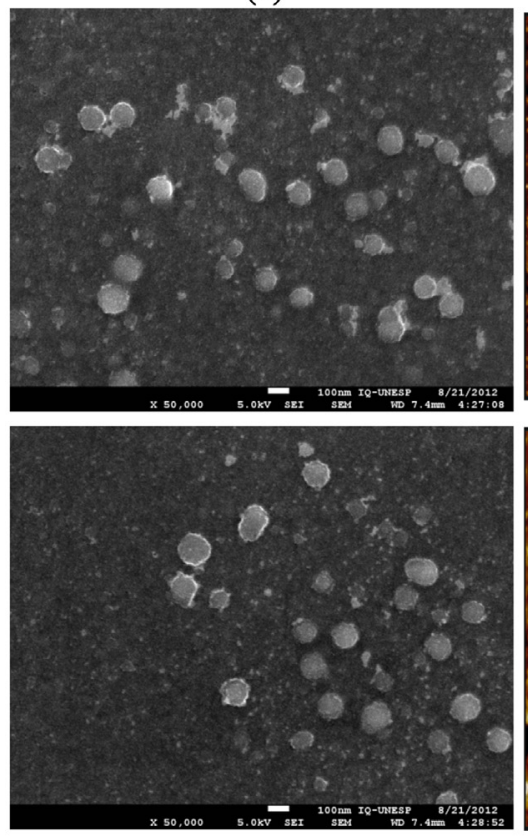

(b)
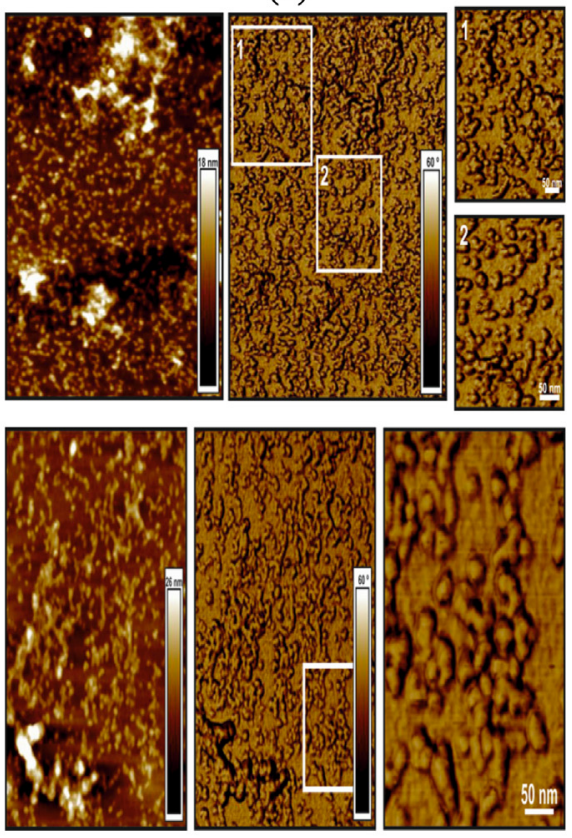

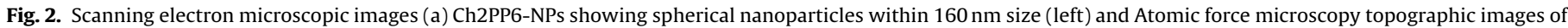
(b) Ch2PP6-NPs showing nanoparticles with $50 \mathrm{~nm}$ (right). 


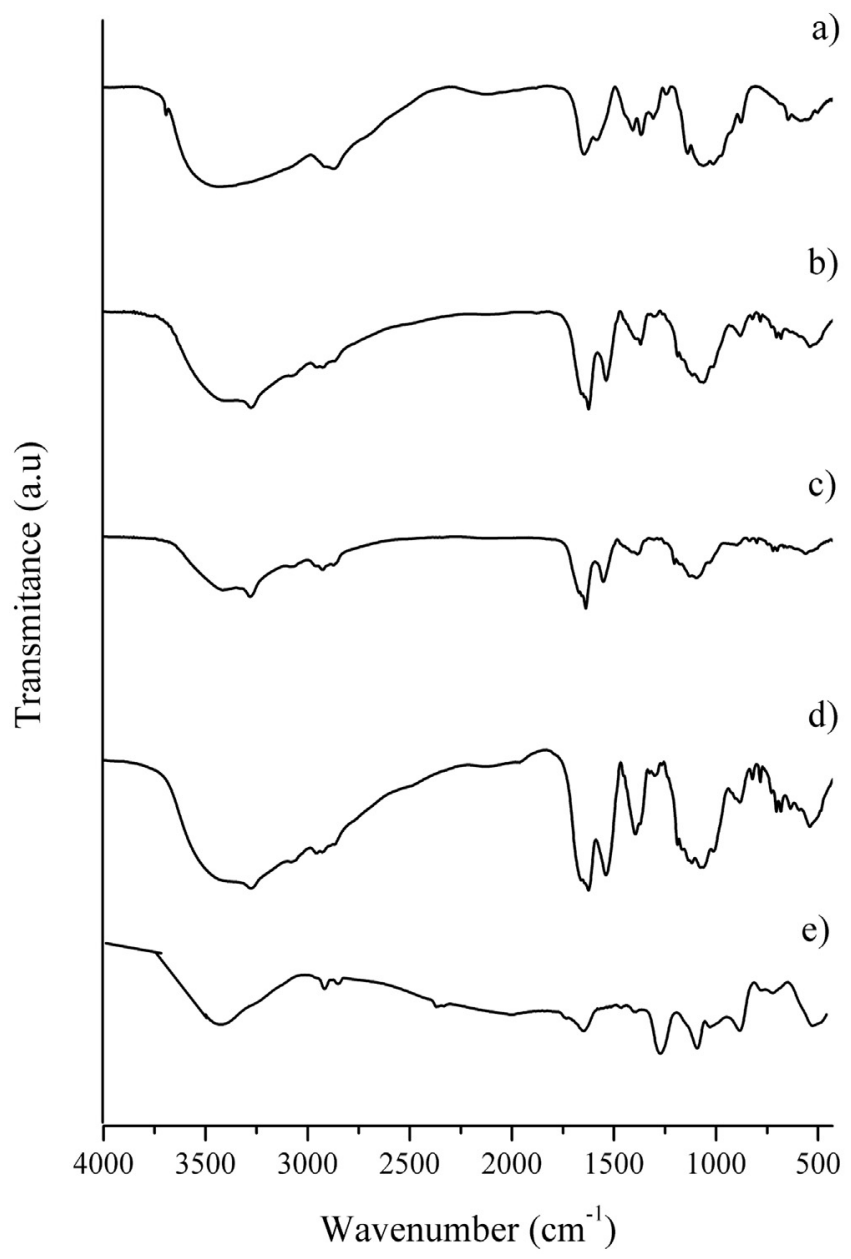

Fig. 3. FTIR spectra of $\mathrm{Ch}$ and of $\mathrm{Ch}-\mathrm{PP}-\mathrm{NPs}$ with various $\mathrm{PP}$ concentrations. (a) Ch; (b) Ch2PP6-NPs, with PP at 0.6\% (w/v); (c) Ch2PP12-NPs, with PP at $1.2 \%(w / v)$ and (d) Ch2PP21-NPs, with PP at 2.1\% (w/v) and PP (e).

$\mathrm{P}-\mathrm{O}-\mathrm{P}$ linkages, respectively, while the $\mathrm{O}-\mathrm{P}-\mathrm{O}$ bending vibration is found at around $490 \mathrm{~cm}^{-1}$.

The nature of the interaction and formation of nanoparticles was evidenced through the FTIR data, when Ch is crosslinked with PP, the nanoparticle formation of Ch-PP-NPs occurs and this interaction was evidenced by amide I groups. The band is unfolds giving rise a new band at $1674 \mathrm{~cm}^{-1}$. The same behavior was observed for the $\mathrm{N}-\mathrm{H}$ bending from amine and amide I, shifting to $1551 \mathrm{~cm}^{-1}$ and the $-\mathrm{CH}_{2}$ bending, shifting to $1408 \mathrm{~cm}^{-1}$. However, the interaction of $\mathrm{PP}$ with $\mathrm{Ch}$ in nanoparticles was not evident through FTIR data. The interaction of $\mathrm{Ch}$ with PP was observed only at the range between 780 and $550 \mathrm{~cm}^{-1}$, this range is characteristic of symmetric stretching modes of these linkages, vs(P-O-P) and $\mathrm{O}-\mathrm{P}-\mathrm{O}$ deformation. These results are not clear if there is variation in the size of PP chain, as well as, the dependence on Ch-PP-NPs nanoparticles. Thus, the careful study by solid state NMR, using the ${ }^{13} \mathrm{C}$ and ${ }^{31} \mathrm{P}$ nuclei with structural probe will confirm the interaction between PP and Ch, as well as, the size of the polyphosphate chain in the future.

The analysis of DSC results shows the influence of the increase on PP concentration on the Ch-PP-NPs formation. Table 3 presents a summary of the temperature transitions and associated enthalpies of each Ch and PP melting peak of the pristine components and investigated nanoparticles produced by ionotropic gelation.

As indicated in Table 3, Ch exhibits a sharp endothermic peak at $75^{\circ} \mathrm{C}$ ascribed to the loss of water adsorbed by $\mathrm{Ch}$ and an exothermic peak at $302^{\circ} \mathrm{C}$ associated with the decomposition of the $\mathrm{Ch}$. Furthermore, the DSC thermogram of PP showed two endothermic
Table 3

Peak temperatures and enthalpy changes in the DSC thermograms collected from CS, PP and nanoparticles obtained with a varying concentration of PP.

\begin{tabular}{llll}
\hline Samples & Onset $/{ }^{\circ} \mathrm{C}$ & $\begin{array}{l}\text { Melting } \\
\text { peak } /{ }^{\circ} \mathrm{C}\end{array}$ & $\begin{array}{l}\text { Enthalpy }(\Delta \mathrm{H}) / \\
\mathrm{J} / \mathrm{g}^{-1}\end{array}$ \\
\hline $\mathrm{Ch}$ & 35.66 & 75.41 & 145.2 \\
& 285.52 & 302.84 & 118.3 \\
PP & 300.6 & 340.54 & 81.27 \\
Ch2PP6-NPs & 39.04 & 80.72 & 225.6 \\
& 239.50 & 249.67 & 26.34 \\
Ch2PP12-NPs & 38.82 & 80.34 & 206.2 \\
Ch2PP21-NPs & 236.26 & 242.77 & 44.57 \\
& 38.78 & 83.49 & 206 \\
& 234.97 & 240.13 & 57.26 \\
\hline
\end{tabular}

peaks associate to a glass transition peak at $271^{\circ} \mathrm{C}$ and an exothermic peak in $351^{\circ} \mathrm{C}$ corresponding to peak assigned to phosphate chains crystallization process that occurs in conjunction with a condensation process (Guinesi \& Cavalheiro, 2006; Kittur, Harish Prashanth, Udaya Sankar, \& Tharanathan, 2002; Mucha \& Pawlak, 2005; Neto et al., 2005).

In the DSC thermograms of Ch2PP6-NPs, Ch2PP12-NPs, Ch2PP21-NPs, the exothermic peaks were shifted toward lower temperature than $\mathrm{PP}$ and $\mathrm{Ch}$ alone. The exothermic peaks for Ch2PP6-NPs appeared at $249^{\circ} \mathrm{C}$ and at $242{ }^{\circ} \mathrm{C}$ for Ch2PP12-NPs and for Ch2PP21-NPs, respectively. These exothermic peaks appeared mainly due to decomposition and dissociation of $\mathrm{Ch}$ and PP hydrolysis, decreasing the thermal stability of the Ch-PP-NPs. The increase in the enthalpy of Ch2PP6-NPs, Ch2PP12-NPs and Ch2PP21-NPs are observed with an increased in phosphate content in the nanoparticles (Fig. 4).

As evidenced from X-Ray Diffraction (XRD) results (data not shown), Ch-PP-NPs showed a pattern predominately amorphous, which corroborates with DSC curves. This finding suggests that PP is dispersed in the matrix of Ch-PP-NPs showing the amorphous nature.

\section{Association efficiency and in vitro cytotoxicity assay}

Considering the results of factorial design, Ch2PP6-NPs was chosen as the best formulation to encapsulate de peptide cRGDfV, since it has the smallest particle size $(166 \mathrm{~nm})$ and the higher ZP value (38.7 mV).

The incorporation of peptide in the Ch2PP6-NPs was achieved by dissolving protein in PP solutions. The peptide loading did not significantly change the size $(233 \mathrm{~nm})$, however it was possible to see an increase in the ZP of the Ch2PP6-NPs ( $46.5 \mathrm{mV})$ after peptide encapsulation. This fact indicates that cRGDfV was conveniently associated within the Ch-PP-NPs.

The AE was 97.0\%, similar results were found for ChTripolyphosphate nanoparticles (Csaba et al., 2009; Dudhani \& Kosaraju, 2010; Hashad, Ishak, Fahmy, Mansour, \& Geneidi, 2016; Liu et al., 2012; Ragelle, Vanvarenberg, Vandermeulen, \& Preat, 2016; Xu \& Du, 2003). This result suggested that PP could be an option for the development of Ch-NPs with high AE instead of using only the trypoliphosphate.

To assess the biocompatibility of Ch2PP6-NPs and cRGDfVloaded-Ch2PP6-NPs, in vitro cell culture was performed and viability was accessed by the resazurin (Alamar Blue ${ }^{\circledR}$ ) reduction assay in Caco-2 cell line. The results of cell viability were compared with those of non-exposed cells (control) and are shown in terms of\% of control. The cell viability above $70 \%$ of the control is an indication of "no toxicity" or of a safe material, and only viability below 70\% considers the material as toxic (Borges et al., 2006; Doktorovova, Souto, \& Silva, 2014; Huang, Khor, \& Lim, 2016). 


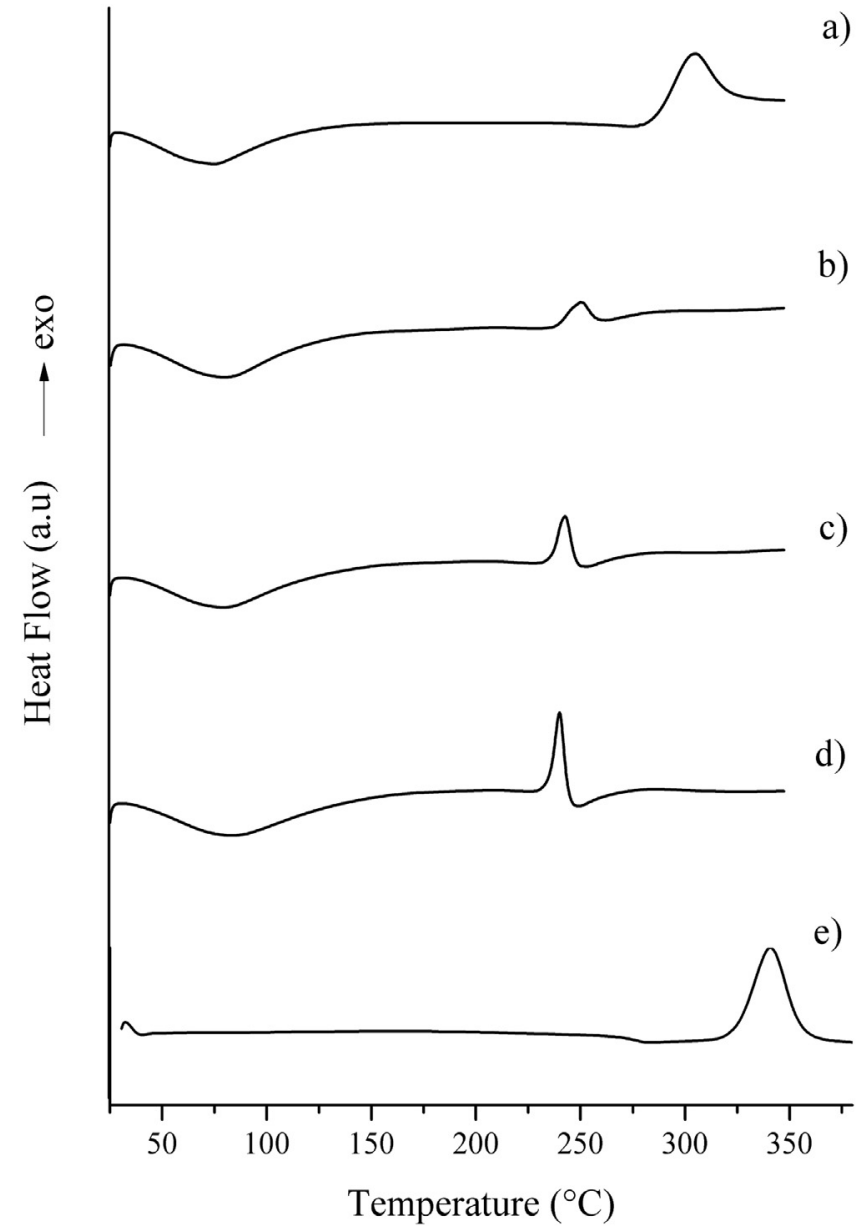

Fig. 4. DSC curves of $\mathrm{Ch}$ and of Ch-PP-NPs with different concentrations of PP. (a) Ch; (b) Ch2PP6-NPs, with PP at $0.6 \%(w / v) ;(c)$ Ch2PP12-NPs, with PP at $1.2 \%(w / v)$ and (d) Ch2PP21-NPs, with PP at 2.1\% (w/v) and PP (e).

Fig. 5 compares the viability of Caco-2 after exposure to Ch2PP6NPs, cRGDfV solution and cRGDfV-loaded-Ch2PP6-NPs $(1,5,10,15$, $20 \mu \mathrm{g} / \mathrm{mL}$ ) for $24 \mathrm{~h}$ and $48 \mathrm{~h}$. No differences on the viability were found between all the treatments, demonstrating that these compounds by themselves did not show any apparent toxicity to the Caco-2 cell line even after long periods of incubation at the same concentration.

Cell viability assessment upon exposure of Caco-2 cells to Ch2PP6-NPs (left set of columns), cRGDfV solution (middle set of columns) and cRGDfV-loaded-Ch2PP6-NPs (left set of columns, cRGDfV-Ch2PP6-NPs), at different concentrations (as indicated by different bas pattern) for $24 \mathrm{~h}$ (left panel) and $48 \mathrm{~h}$ (right panel). Cell viability was assessed by Alamar Blue assay and data is shown in percentage of control (non-exposed cells).

These studies showed that $\mathrm{Ch}$ and $\mathrm{PP}$ are an attractive combination for development of nanoparticles for protein/peptide delivery due to various characteristics including its biocompatibility, low toxicity, and low immunogenicity. These results clearly indicate that all materials don't have any cytotoxic effect on Caco-2 cells and they are highly nontoxic and biocompatible for protein/peptide delivery.

\section{Conclusion}

Experimental factorial design enables to successfully formulate Ch-PP NPs with suitable physicochemical parameters requiring a minimum of experiments. The influence of the independent vari-

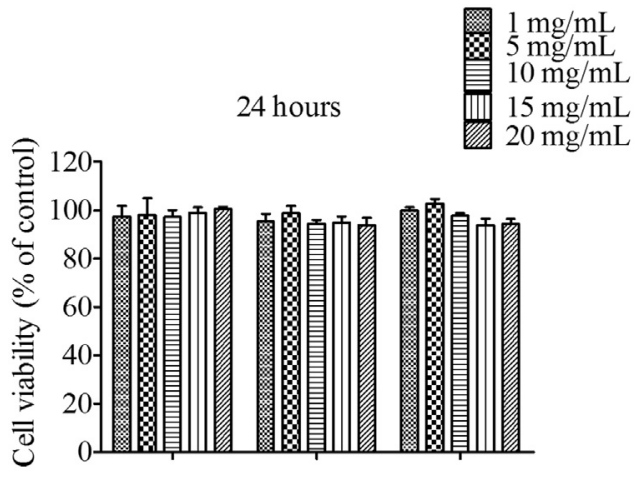

48 hours

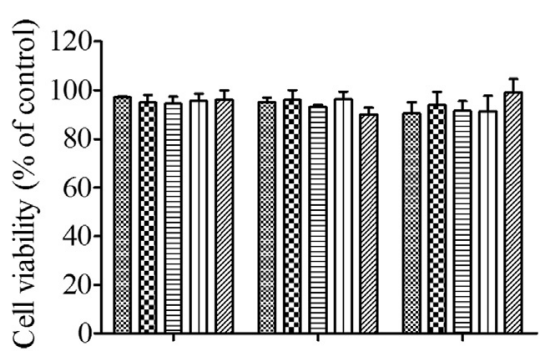

Fig. 5. Cell viability of the cRGDfV-loaded in Ch2PP6-NPs by Alamar Blue ${ }^{\circledast s}$ assay for cell proliferation activity in vitro in Caco- 2 cells ${ }^{*} p<0.05$.

Cell viability assessment upon exposure of Caco-2 cells to Ch2PP6-NPs (left set of columns), cRGDfV solution (middle set of columns) and cRGDfV-loaded-Ch2PP6-NPs (left set of columns, cRGDfV-Ch2PP6-NPs), at different concentrations (as indicated by different bas pattern) for $24 \mathrm{~h}$ (left panel) and $48 \mathrm{~h}$ (right panel). Cell viability was assessed by Alamar Blue assay and data is shown in percentage of control (nonexposed cells).

ables (i.e., concentration of $\mathrm{Ch}$ and PP) on the Z-Ave, PdI and ZP was analyzed and showed that the size of NPs are highly dependent on the Ch concentrations. Optimal parameters were obtained for formulation Ch2PP6-NPs composed of $2.0 \%(\mathrm{w} / \mathrm{v})$ of $\mathrm{Ch}$ and $0.6 \%$ $(\mathrm{w} / \mathrm{v})$ of PP. For these conditions, the average size of particles were $166 \mathrm{~nm}$, zeta potential was $38.7 \mathrm{mV}$. The presented SEM/AFM, FTIR and DSC analysis confirmed the Ch-PP-NPs formation by morphology evaluation, interaction between components of particles and thermal behavior, respectively. In the SEM/AFM studies, results showed a spherical shape of the Ch2PP6-NPs with a homogeneous distribution. FTIR and DSC analysis demonstrated the interaction and partial miscibility between $\mathrm{Ch}$ and PP indicating the formation of the Ch-PP-NPs. Additionally, the cytotoxicity assay showed that Ch2PP6-NPs and cRGDfV-loaded Ch2-PP6-NPs did not affect, or affected on a low level, the cell viability, demonstrating none or very low toxicity in Caco- 2 cell line, indicating that the developed nanoparticles are biocompatible and suitable for protein/peptide delivery systems.

\section{Acknowledgments}

This work was supported by FAPESP (number 2012-10174-3), Brazil, São Paulo. Moreover, we are grateful to the 'MacrobehaviorMesostructure-Nanotechnology' SGIker unit of the UPV/EHU. We also acknowledge FEDER/COMPETE/POCI (POCI-01-0145FEDER-006958) and FCT-Portuguese Foundation for Science and Technology (to AMS).

\section{References}

Andreani, T., Kiill, C. P., de Souza, A. L., Fangueiro, J. F., Fernandes, L., Doktorovova, S., ... \& Silva, A. M. (2014). Surface engineering of silica nanoparticles for oral insulin delivery: Characterization and cell toxicity studies. Colloids and Surfaces B: Biointerfaces, 123, 916-923. 
Andreani, T., de Souza, A. L., Kiill, C. P., Lorenzon, E. N., Fangueiro, J. F., Calpena, A C., ... \& Souto, E. B. (2014). Preparation and characterization of PEG-coated silica nanoparticles for oral insulin delivery. International Journal of Pharmaceutics, 473(1-2), 627-635.

Barbi, M. D. S., Carvalho, F. C., Kiill, C. P., Da Silva Barud, H., Santagneli, S. H., Ribeiro, S. J. L., \& Gremião, M. P. D. (2015). Preparation and characterization of chitosan nanoparticles for zidovudine nasal delivery? Journal of Nanoscience and Nanotechnology, 15(1), 865-874.

Bluebond-Langner, M., Perkel, D., Goertzel, T., Nelson, K., \& McGeary, J. (1990). Children's knowledge of cancer and its treatment: Impact of an oncology camp experience? Journal of Pediatrics, 116(2), 207-213.

Borges, O., Cordeiro-da-Silva, A., Romeijn, S. G., Amidi, M., de Sousa, A., Borchard, G., \& Junginger, H. E. (2006). Uptake studies in rat Peyer's patches, cytotoxicity and release studies of alginate coated chitosan nanoparticles for mucosal vaccination. Journal of Controlled Release, 114(3), 348-358.

Brow, R. K., Tallant, D. R., Myers, S. T., \& Phifer, C. C. (1995). The short-range structure of zinc polyphosphate glass. Journal of Non-Crystalline Solids, 191(1-2), 45-55

Brow, R. K. (2000). Review: The structure of simple phosphate glasses. Journal of Non-Crystalline Solids, 263-264, 1-28

Callens, C., \& Remon, J. P. (2000). Evaluation of starch-maltodextrin-Carbopol 974 P mixtures for the nasal delivery of insulin in rabbits? Journal of Controlled Release, 66(2-3), 215-220.

Calvo, P., Remuñán-López, C., Vila-Jato, J. L., \& Alonso, M. J. (1997). Novel hydrophilic chitosan-polyethylene oxide nanoparticles as protein carriers. Journal of Applied Polymer Science, 63(1), 125-132.

Casettari, L., Cespi, M., Palmieri, G. F., \& Bonacucina, G. (2013). Characterization of the interaction between chitosan and inorganic sodium phosphates by means of rheological and optical microscopy studies. Carbohydrate Polymer, 91(2), 597-602.

Chandra Hembram, K., Prabha, S., Chandra, R., Ahmed, B., \& Nimesh, S. (2016). Advances and characterization of chitosan nanoparticles for therapeutics. Artif Cells Nanomed Biotechnol, 44(1), 305-314 (in preparation).

Chen, F., Zhang, Z. R., \& Huang, Y. (2007). Evaluation and modification of $\mathrm{N}$-trimethyl chitosan chloride nanoparticles as protein carriers? International Journal of Pharmaceutics, 336(1), 166-173.

Csaba, N., Koping-Hoggard, M., \& Alonso, M. J. (2009). Ionically crosslinked chitosan/tripolyphosphate nanoparticles for oligonucleotide and plasmid DNA delivery? International Journal of Pharmaceutics, 382(1-2), 205-214.

de Moura, M. R., Aouada, F. A., Avena-Bustillos, R. J., McHugh, T. H., Krochta, J. M., \& Mattoso, L. H. C. (2009). Improved barrier and mechanical properties of novel hydroxypropyl methylcellulose edible films with chitosan/tripolyphosphate nanoparticles? Journal of Food Engineering, 92(4), 448-453.

de Pinho Neves, A. L., Milioli, C. C., Müller, L., Riella, H. G., Kuhnen, N. C., \& Stulzer, H. K. (2014). Factorial design as tool in chitosan nanoparticles development by ionic gelation technique. Colloids and Surfaces A: Physicochemical and Engineering Aspects, 445, 34-39.

Doktorovova, S., Souto, E. B., \& Silva, A. M. (2014). Nanotoxicology applied to solid lipid nanoparticles and nanostructured lipid carriers - A systematic review of in vitro data? European Journal of Pharmaceutics and Biopharmaceutics, 87(1), $1-18$.

Dudhani, A. R., \& Kosaraju, S. L. (2010). Bioadhesive chitosan nanoparticles: Preparation and characterization. Carbohydrate Polymers, 81(2), 243-251.

Fabregas, A., Minarro, M., Garcia-Montoya, E., Perez-Lozano, P., Carrillo, C., Sarrate, R., .. \& \& Sune-Negre, J. M. (2013). Impact of physical parameters on particle size and reaction yield when using the ionic gelation method to obtain cationic polymeric chitosan-tripolyphosphate nanoparticles. International Journal of Pharmaceutics, 446(1-2), 199-204.

Fan, W., Yan, W., Xu, Z., \& Ni, H. (2012). Formation mechanism of monodisperse, low molecular weight chitosan nanoparticles by ionic gelation technique. Colloids and Surfaces B: Biointerfaces, 90, 21-27.

Fundueanu, G., Constantin, M., Dalpiaz, A., Bortolotti, F., Cortesi, R., Ascenzi, P., \& Menegatti, E. (2004). Preparation and characterization of starch/cyclodextrin bioadhesive microspheres as platform for nasal administration of Gabexate Mesylate (Foy) in allergic rhinitis treatment. Biomaterials, 25(1), 159-170.

Gan, Q., Wang, T., Cochrane, C., \& McCarron, P. (2005). Modulation of surface charge, particle size and morphological properties of chitosan-TPP nanoparticles intended for gene delivery. Colloids and Surfaces B: Biointerfaces, 44(2-3), 65-73.

Gavini, E., Rassu, G., Sanna, V., Cossu, M., \& Giunchedi, P. (2005). Mucoadhesive microspheres for nasal administration of an antiemetic drug, metoclopramide: In-vitro/ex-vivo studies. Journal of Pharmacy and Pharmacology, 57(3), 287-294.

Gavini, E., Hegge, A. B., Rassu, G., Sanna, V., Testa, C., Pirisino, G., . . . \& Giunchedi, P. (2006). Nasal administration of carbamazepine using chitosan microspheres: In vitro/in vivo studies. International Journal of Pharmaceutics, 307(1), 9-15.

Guinesi, L. S., \& Cavalheiro, É. T. G. (2006). The use of DSC curves to determine the acetylation degree of chitin/chitosan samples. Thermochimica Acta, 444(2), 128-133.

Hasanovic, A., Zehl, M., Reznicek, G., \& Valenta, C. (2009).

Chitosan-tripolyphosphate nanoparticles as a possible skin drug delivery system for aciclovir with enhanced stability. Journal of Pharmacy and Pharmacology, 61(12), 1609-1616.

Hashad, R. A., Ishak, R. A., Fahmy, S., Mansour, S., \& Geneidi, A. S. (2016). Chitosan-tripolyphosphate nanoparticles: Optimization of formulation parameters for improving process yield at a novel $\mathrm{pH}$ using artificial neural networks. International Journal of Biological Macromolecules, 86, 50-58.

Hu, B., Pan, C., Sun, Y., Hou, Z., Ye, H., \& Zeng, X. (2008). Optimization of fabrication parameters to produce chitosan-tripolyphosphate nanoparticles for delivery of tea catechins? Journal of Agricultural and Food Chemistry, 56(16), 7451-7458.

Huang, H., \& Yang, X. (2004). Synthesis of chitosan-stabilized gold nanoparticles in the absence/presence of tripolyphosphate. Biomacromolecules, 5(6), 2340-2346.

Huang, M., Khor, E., \& Lim, L.-Y. (2016). Uptake and cytotoxicity of chitosan molecules and nanoparticles: Effects of molecular weight and degree of deacetylation. Pharmaceutical Research, 21(2), 344-353.

Hudgens, J. J., Brow, R. K., Tallant, D. R., \& Martin, S. W. (1998). Raman spectroscopy study of the structure of lithium and sodium ultraphosphate glasses. Journal of Non-Crystalline Solids, 223(1-2), 21-31.

Jain, A., Thakur, K., Sharma, G., Kush, P., \& Jain, U. K. (2016). Fabrication, characterization and cytotoxicity studies of ionically cross-linked docetaxel loaded chitosan nanoparticles. Carbohydrate Polymers, 137, 65-74.

Jiang, H., Wu, H., Xu, Y. L., Wang, J. Z., \& Zeng, Y. (2011). Preparation of galactosylated chitosan/tripolyphosphate nanoparticles and application as a gene carrier for targeting SMMC7721 cells. Journal of Bioscience and Bioengineering, 111(6), 719-724.

Jin, H., Pi, J., Yang, F., Wu, C., Cheng, X., Bai, H., . . \& \& Chen, Z. W. (2016). Ursolic acid-loaded chitosan nanoparticles induce potent anti-angiogenesis in tumor. Applied Microbiology and Biotechnology.

Jonassen, H., Kjoniksen, A. L., \& Hiorth, M. (2012). Stability of chitosan nanoparticles cross-linked with tripolyphosphate. Biomacromolecules, 13(11), 3747-3756.

Joseph, J. J., Sangeetha, D., \& Gomathi, T. (2016). Sunitinib loaded chitosan nanoparticles formulation and its evaluation. International Journal of Biological Macromolecules, 82, 952-958.

Khawaja, E. E., Durrani, S. M. A., Al-Adel, F. F., Salim, M. A., \& Hussain, M. S. (2016). $\mathrm{X}$-ray photoelectron spectroscopy and Fourier transform-infrared studies of transition metal phosphate glasses. Journal of Materials Science, 30(1), 225-234.

Kittur, F. S., Harish Prashanth, K. V., Udaya Sankar, K., \& Tharanathan, R. N. (2002). Characterization of chitin, chitosan and their carboxymethyl derivatives by differential scanning calorimetry. Carbohydrate Polymers, 49(2), 185-193.

Koukaras, E. N., Papadimitriou, S. A., Bikiaris, D. N., \& Froudakis, G. E. (2012). Insight on the formation of chitosan nanoparticles through ionotropic gelation with tripolyphosphate? Molecular Pharmaceutics, 9(10), 2856-2862.

Liu, G., Xu, D., Jiang, M., \& Yuan, W. (2012). Preparation of bioactive interferon alpha-loaded polysaccharide nanoparticles using a new approach of temperature-induced water phase/water-phase emulsion. International Journal of Nanomedicine, 7, 4841-4848.

Montha, W., Maneeprakorn, W., Buatong, N., Tang, I. M., \& Pon-On, W. (2016). Synthesis of doxorubicin-PLGA loaded chitosan stabilized (Mn: Zn)Fe2O4 nanoparticles: Biological activity and $\mathrm{pH}$-responsive drug release. Materials Science E Engineering C: Materials for Biological Applications, 59, 235-240.

Moustafa, Y. M., \& El-Egili, K. (1998). Infrared spectra of sodium phosphate glasses. Journal of Non-Crystalline Solids, 240(1-3), 144-153.

Mucha, M., \& Pawlak, A. (2005). Thermal analysis of chitosan and its blends. Thermochimica Acta, 427(1-2), 69-76.

Neto, C. G. T., Giacometti, J. A., Job, A. E., Ferreira, F. C., Fonseca, J. L. C., \& Pereira, M R. (2005). Thermal analysis of chitosan based networks? Carbohydrate Polymers, 62(2), 97-103.

Pickup, D. M., Newport, R. J., Barney, E. R., Kim, J. Y., Valappil, S. P., \& Knowles, J. C. (2014). Characterisation of phosphate coacervates for potential biomedical applications. Journal of Biomaterials Applications, 28(8), 1226-1234.

Ragelle, H., Vanvarenberg, K., Vandermeulen, G., \& Preat, V. (2016). Chitosan nanoparticles for SiRNA delivery In vitro. Methods in Molecular Biology, 1364, $143-150$.

Sadeghi, A. M., Dorkoosh, F. A., Avadi, M. R., Saadat, P., Rafiee-Tehrani, M., \& Junginger, H. E. (2008). Preparation, characterization and antibacterial activities of chitosan, N-trimethyl chitosan (TMC) and N-diethylmethyl chitosan (DEMC) nanoparticles loaded with insulin using both the ionotropic gelation and polyelectrolyte complexation methods. International Journal of Pharmaceutics, 355(1-2), 299-306.

Wang, W., Tong, C. Y., Liu, B., Liu, X. Y., \& Li, T. (2015). Preparation and functional characterization of tumor-targeted folic acid-chitosan conjugate nanoparticles loaded with mitoxantrone. Journal of Controlled Release, 213, e110-111.

Xu, Y., \& Du, Y. (2003). Effect of molecular structure of chitosan on protein delivery properties of chitosan nanoparticles. International Journal of Pharmaceutics, 250(1), 215-226

Yang, W., Fu, J., Wang, T., \& He, N. (2009). Chitosan/sodium tripolyphosphate nanoparticles: Preparation, characterization and application as drug carrier. Journal of Biomedical Nanotechnology, 5(5), 591-595. 